\title{
Fluid-structure interactions with applications to biology
}

\author{
Wei-Xi Huang ${ }^{1} \cdot$ Silas Alben $^{2}$
}

Published online: 2 November 2016

(c) The Chinese Society of Theoretical and Applied Mechanics; Institute of Mechanics, Chinese Academy of Sciences and Springer-Verlag Berlin Heidelberg 2016

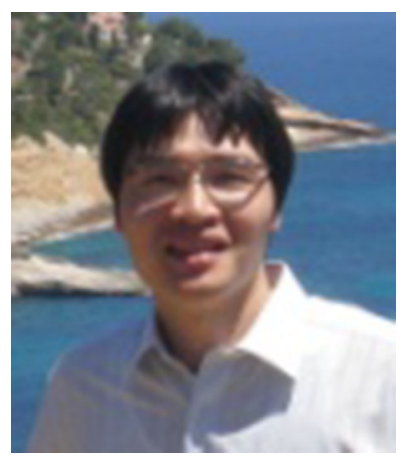

Wei-Xi Huang received his bachelor's degree and master's degree from Tsinghua University in 2001 and 2004, respectively, and he completed his Ph.D. at Korea Advanced Institute of Science and Technology (KAIST) in 2009. In 2010, Huang entered the School of Aerospace Engineering of Tsinghua University as a lecturer, where he is now serving as an associate professor (2012-present). Huang's current research focus is on the numerical study of turbulence control and development of novel schemes for turbulent drag reduction. Huang is also interested in computational biofluid mechanics. He has been developing computational methods for fluid-flexible body interactions, with the goal of simulating and obtaining physical insight into problems from biomechanics. Huang received China National Science Funds for Excellent Young Scientists in 2013. Huang is currently a member of the International Exchange and Cooperation Committee of the Chinese Society of Theoretical and Applied Mechanics, the American Physical Society, and the European Mechanics Society.

Wei-Xi Huang

hwx@tsinghua.edu.cn

Silas Alben

alben@umich.edu

1 Department of Engineering Mechanics, Tsinghua University, Beijing 100084, China

2 Department of Mathematics, University of Michigan, Ann Arbor, MI, USA 


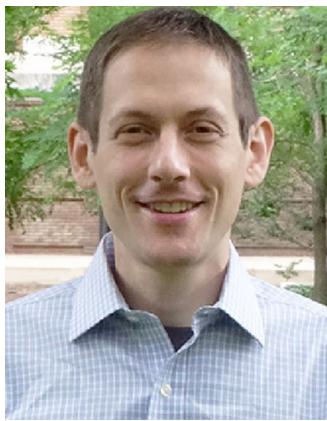

Silas Alben received his bachelor's degree from Harvard University in 1999 and his master's degree and Ph.D. from the Courant Institute at New York University in 2002 and 2004, respectively. Following a postdoc at Harvard and an assistant professorship at Georgia Institute of Technology (Georgia Tech), he joined the Department of Mathematics at the University of Michigan in 2012 as an associate professor. Professor Alben is an applied mathematician working in fluid and solid mechanics, often inspired by biology. One area of focus is the locomotion of organisms in fluid and terrestrial environments, including swimming fish and slithering snakes. This work uses a combination of mathematical modelling, theoretical analysis, and development of numerical methods. Alben is the recipient of a Sloan Fellowship and funding from the USA National Science Foundation and Air Force Office of Scientific Research.

Fluid-structure interactions (FSI) are encountered by almost all animals and plants that live in fluid environments, e.g. flying insects/birds, swimming fish, blood cells, seeds, leaves, and trees. The interactions of living species with fluids are commonplace in our daily life and have attracted wide attention for a long time. However, they are still puzzling today for their intrinsic complexities, which bring major challenges in experimental, theoretical, and numerical studies. In such systems, the biological structures can be deformed under the forces exerted by the surrounding fluid. On the other hand, the deformable structures act on the fluid, forcing it to move with the moving boundary. Together, the interaction between the fluid and the biological structures can allow for efficient biological locomotion, which arose gradually during a long evolutionary history. Therefore, new insights into FSI mechanisms may help us not only to resolve enduring puzzles, but also to improve our designs in biological and biomechanical applications.

Significant progress in FSI research related to natural biological phenomena during the last two decades can be found in the literature. Attributed to the rapid developments in experimental measurements and numerical techniques, the non-linear behaviors in both the fluid and biological structures and their mutual coupling can be captured. As a result, some new findings have been reported, and as a few representative examples among them, the hysteresis of a massive slender body flapping in viscous fluid was disclosed; a deep understanding of the relation between the active and passive flapping motions (swimming fish versus flapping flags) was obtained; different interaction modes of upstream shed vortices with downstream swimming bodies were detected, which may impede or assist the propulsion of the downstream bodies; fluttering, tumbling, and chaotic motions of falling leaves were observed and were also shown to be illustrative of lift generation in insect flight; tank-treading and tumbling modes of a blood cell in a shear flow were identified by experimental visualization, theoretical modeling, and numerical simulation. These new findings attracted broad interest and lively discussions. Nevertheless, further investigations on related topics are necessary. For instance, a first-principles explanation of hysteresis phenomena in fluid-flexible body interactions is still lacking; the role of the passive response in active swimming and flying problems is worth further discussion; extensions of FSI models to more complex and real configurations remains challenging.

This thematic issue consists of five articles contributed by experts who are active on related topics. Two numerical models, i.e., active/passive flapping motion and cell deformation, are mainly considered in these articles, where we can see the state-of-the-art developments on numerical modeling of FSI with applications to biology. Wang et al. [1] give a comprehensive review of recent work on selfpropelled mechanical systems powered by flapping motion; Ryu et al. [2] study the ground effect on a flexible propulsor with active flapping head; Yeh and Alexeev [3] find that the propulsive efficiency can be improved by attaching a passive part to an internally actuated (active flapping) flexible plate. On the other hand, Peng et al. [4] establish a computational model of a lipid bilayer membrane for simulation of vesicle/cell deformation subjected to fluid shear, as a prototype for understanding the biophysics underlying mechanosensation, which is an important biological FSI process; Wei et al. [5] carry out a numerical study on continuous sorting of flexible cells by using the deterministic lateral displacement (DLD) method. We hope that these studies will bring new interests and inspirations to this field.

Finally, we appreciate the efforts made by all the contributors for this thematic issue. We are grateful to the former Editor-in-Chief of Acta Mechanica Sinica, Prof. Quan-Shui Zheng, for inviting us as the guest editors of this thematic issue. We also thank the staff of the editorial office of Acta Mechanica Sinica for managing, assistance, and support.

\section{References}

1. Wang, S., He, G., Zhang, X.: Self-propulsion of flapping bodies in viscous fluids: recent advances and perspectives. Acta Mech. Sin. (2016). doi:10.1007/s10409-016-0578-y 
2. Ryu, J., Park, S.G., Kim, B., et al.: Flapping dynamics of a flexible propulsor near ground. Acta Mech. Sin. (2016). doi:10.1007/ s10409-016-0571-5

3. Yeh, P.D., Alexeev, A.: Biomimetic flexible plate actuators are faster and more efficient with a passive attachment. Acta Mech. Sin. (2016). doi:10.1007/s10409-016-0592-0
4. Peng, Z., Pak, O.S., Liu, A.P., et al.: On the gating of mechanosensitive channels by fluid shear stress. Acta Mech. Sin. (2016). doi:10. 1007/s10409-016-0606-y

5. Wei, Q., Xu, Y.-Q., Tang, X.-Y., et al.: An IB-LBM study of continuous cell sorting in deterministic lateral displacement arrays. Acta Mech. Sin. (2016). doi:10.1007/s10409-016-0566-2 